\title{
Historical perspective of laser beam shaping
}

\author{
David L. Shealy* \\ University of Alabama at Birmingham \\ Department of Physics, $15303^{\text {rd }}$ Avenue South, CH310 \\ Birmingham, AL 35294-1170 USA
}

\begin{abstract}
An overview of the history and current practices of laser beam shaping is presented. When diffraction effects are not important, geometrical methods for laser beam shaping (ray tracing, conservation of energy within a bundle of rays, and the constant optical path length condition) can be used to determine system configurations, including aspheric elements and spherical-surface GRIN lenses, which are required to transform an input laser beam profile into a more useful form of illumination. This paper also summarizes applications of these techniques to the optical design of a two-planoaspheric lens system for shaping a rotationally symmetric Gaussian beam, a two-mirror system with no central obscuration for shaping an elliptical Gaussian input beam, and a three-element spherical surface GRIN system for shaping a rotationally symmetric Gaussian beam.
\end{abstract}

Keywords: laser; beaming shaping; geometrical optics; optical design; irradiance mapping

\section{INTRODUCTION}

Early thoughts of beam shaping in non-laser systems can be traced to before the days of Archimedes and his burning glass, ${ }^{1,2}$ where optics was reported to concentrate -- to increase the power density of -- solar radiation. The literature is rich with reports of various optical systems used as solar collectors. ${ }^{3,4,5,6,7}$ Welford and Winston ${ }^{8}$ have presented a good accounting of non-imaging (non-focusing) optics used as solar collectors, including an ideal light collector, 910 which concentrates a beam by the maximum amount allowed by phase space considerations. Burkhard and Shealy ${ }^{11}$ have used a differential equation method to design a reflecting surface, which distributes the irradiance over a receiver surface in a prescribed manner. McDermit and Horton ${ }^{12,13}$ presented a generalized technique for designing a rotationally symmetric reflective solar collector, which can heat the collector surface in a prescribed manner. Beam shaping has also been used in opto-electronics to achieve maximum power transfer between a micro-optics light source and an optical fiber, ${ }^{14,15}$ in radiative heat transfer, ${ }^{16,17,18}$ in illumination applications, ${ }^{19,20,21,22}$ and for reflector synthesis. ${ }^{23,24}$

For illumination applications using a laser beam, such as in holography, materials processing, and lithography, it is

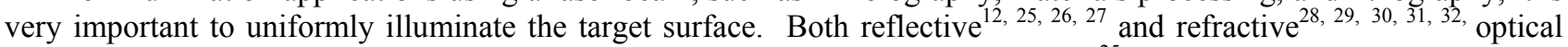
systems have been used to shape laser beam intensity profiles. McDermit and Horton ${ }^{25}$ use conservation of energy within a bundle of rays to design rotationally symmetric reflective optical systems for illuminating a receiver surface in a prescribed manner using a non-uniform input beam profile. Malyak, ${ }^{26}$ Shealy and $\mathrm{Chao}^{27}$ have designed a two-mirror laser profile shaping system with rectangular symmetry and no central obscuration. Kreuzer ${ }^{29}$ has patented a coherentlight optical system using two aspherical surfaces to yield an output beam of desired intensity distribution and wavefront shape. Rhodes and Shealy ${ }^{30}$ derived a set of differential equations using intensity mapping and the constant optical path length condition to calculate the shape of two-aspherical surfaces of a lens system that expands and converts a Gaussian laser beam profile into a collimated, uniform irradiance output beam. Using their method, two-plano-aspherical lenses have been designed, fabricated and used for laser beam shaping in a holographic projection system. ${ }^{33,34,35}$ Hoffnagle and Jefferson $^{32}$ introduced convex aspherical surfaces for ease of fabrication and a continuous roll-off of the output beam profile for more control of the far-field diffraction pattern into their design of a refractive laser beam shaping system.

Optical design of laser beam shaping systems has evolved considerably from the early work of Frieden ${ }^{28}$ and Kreuzer $^{29}$ during the 1960 's to the contemporary work of many summarized in Refs. 36, 37, and 38. This early work articulated well the goals of some contemporary laser beam shaping applications. Namely, Frieden and Kreuzer sought to define an optical system that would transform an input plane wave with a Gaussian irradiance profile into an output plane wave with uniform irradiance. Conservation of energy along a bundle of rays was used to establish a non-linear mapping of the ray coordinates between the input and output planes. Frieden shows that the phase of the beam over the

* Other author information: E-mail: dls@uab.edu ; Telephone: 205-934-8068; Fax: 205-934-8042. 
output plane may vary by $20 \lambda$ after redistribution of the beam irradiance. Therefore, for laser beam shaping applications when the output beam phase is important, a second optical element must correct phase distortions introduced by the irradiance redistribution. Frieden computed the shaping of an aspherical refracting surface that would re-collimate the output beam parallel to the optical axis and also to the input beam. Keuzer imposed the constant optical path length condition for all rays passing through the beam shaping optics to control phase variation of the output beam. Unfortunately, optical design and fabrication technologies were generally not adequate until the 1980's to permit realistic design, analysis, fabrication, and testing of laser beam shaping systems.

Today, beam shaping is the process of redistributing the irradiance and phase of a beam of optical radiation. The irradiance distribution defines the beam profile, such as, Gaussian, multimode, annular, rectangular, or circular. The phase of the output beam determines its propagation properties. Contemporary laser beam shaping systems can be grouped into two functional categories: field mappers and beam integrators. A field mapper transforms a known input beam into a desired output beam in a prescribed manner, can be effectively lossless, and works well for single-mode beams. A beam integrator breaks the input beam into a large number of facets by a lens-array, and then, tries to spread the energy within each facet over the output region. The output beam profile is a sum of the diffraction patterns of each lens-array aperture. Beam integrators work well for multimode beams where the input profile may be unknown.

Optical design of beam shaping systems can be achieved using either physical or geometrical optics. There is no single beam shaping method that can be used for all applications. Guidance in choosing a beam shaping technique is discussed in Chapter 1 of Ref. 37. For single-mode Gaussian beams calculating the parameter $\beta$ will help determine the quality of solution available and whether geometrical or physical optics methods should be used

$$
\beta=\frac{2 \sqrt{2 \pi} r_{0} Y_{0}}{f \lambda}
$$

where $\lambda$ is the wavelength, $\mathrm{r}_{0}$ is the beam radius or waist, $\mathrm{Y}_{0}$ is half-width of the desired output dimension, and $f$ is the focal length of the focusing optic, or the working distance from the optical system to the target plane for systems without a defined focusing optics. For simple output geometries, such as, circles and rectangles, the following rules of thumb have been developed: ${ }^{39}$

- If $\beta<4$, a beam shaping system will not produce acceptable results.

- When $4<\beta<32$, diffraction effects are significant and should be part of design of beam shaping systems.

- When $\beta>32$, geometrical methods should be adequate for design of beam shaping systems.

This paper will summarize the historical development and application of the geometrical methods ${ }^{40,41}$ for designing reflective and refractive field mapping systems. Field mapping is basic to all beam shaping, since one seeks to design a set of optical elements that map an input field into a desired output optical field.

A historical perspective of the theory of geometrical methods for design of a laser beam shaping system is presented in section 2. A brief overview is presented of the optical design process of incorporating the geometrical optics intensity law for propagation of a bundle of rays and the constant optical path length condition into the ray trace equations, and then, of determining the geometrical contour of several surfaces so that the beam shaping design conditions are satisfied. Optimization-based techniques, such as, genetic algorithms (GA), have also been shown to be effective methods for design of laser beam shaping systems. ${ }^{42}$ Three applications - a two-plano-aspheric lens system for shaping a rotationally symmetric Gaussian beam, a two-mirror system with no central obscuration for shaping an elliptical Gaussian input beam, and a three-element GRIN system with spherical surfaces for shaping a rotationally symmetric Gaussian beam are discussed. These applications have been selected to illustrate how the geometrical methods for optical design of laser beam shaping systems are applied to a range of configurations. Section 3 presents a brief introduction to the benefits of using beam shaping optics when the overall efficiency increases if the detector is uniformly illuminated.

\section{GEOMETRICAL METHODS}

In order to optimize the irradiance within an optical system, the optical field must be determined throughout the system. The optical field is a local plane wave solution of Maxwell's equations or the scalar wave equation. ${ }^{43,44}$ For an isotropic, non-conducting, charge-free medium, the optical field may be written as:

$$
u(\mathbf{r})=u_{0}(\mathbf{r}) \exp \left[i k_{0} S(\mathbf{r})\right]
$$


where $\mathrm{k}_{0}=\omega / \mathrm{c}=2 \pi / \lambda_{0}$ is the wave number in free space; $\mathrm{u}_{0}(\mathbf{r})$ and $\mathrm{S}(\mathbf{r})$ are unknown functions of $\mathbf{r}$. Requiring $\mathrm{u}(\mathbf{r})$ from Eq. (2) satisfy the scalar wave equation leads to the following conditions which must be satisfied:

$$
\begin{gathered}
(\nabla S)^{2}=n^{2} \\
2 u_{0} \nabla S \cdot \nabla u_{0}+u_{0}^{2} \nabla^{2} S=\nabla \cdot\left(u_{0}^{2} \nabla S\right)=\nabla \cdot\left(u_{0}^{2} n \mathbf{a}\right)=\nabla \cdot(I \mathbf{a})=0
\end{gathered}
$$

where $\mathrm{n}$ is the index of refraction, and $I$ is the energy density of the field times the speed of propagation within medium. Equation (3) is known as the eikonal equation and is a basic equation of geometrical optics. The surfaces $\mathrm{S}(\mathrm{x}, \mathrm{y}, \mathrm{z})=$ const. are constant phase fronts of the optical field, have a constant optical path length (OPL) from the source or reference surface, and are known as the geometrical wavefront. Equation (4) expresses conservation of radiant energy within a bundle of rays and is known as the geometrical optics intensity law for propagation of energy. According to geometrical optics, the phase and amplitude of the optical field are evaluated independently. First, the ray paths are evaluated throughout the optical system with ray tracing. Then, the phase of the optical field is computed from the optical path length of the rays passing through the system. The amplitude (or intensity) of the optical field is computed from the density of rays at any point within the system by monitoring the intensity variations along each ray. ${ }^{21,45,46}$

For beam shaping systems with collimated input and output beams as illustrated in Figure 1, a useful expression for the energy within a bundle of rays ${ }^{47}$ as it passes through the system follows by integrating Eq. (4) over reference planes (or wavefront) normal to the input and output beam and then applying Gauss' theorem

$$
I_{\text {in }} d w=I_{\text {out }} d W .
$$

Equation (5) expresses conservation of energy along a bundle of rays between input element of area and the corresponding output element of area on the wavefront or the reference planes normal to the beam. Equation (5) says that the intensity times the cross-sectional area of the beam is constant along the beam as it propagates through the optical system and is a basic equation used for the optical design of laser beam shaping systems. For some beam shaping configurations, it is necessary to introduce the conservation of energy condition into the optical design by using reference surfaces, such as for detectors, which are curved and/or have an arbitrary orientation with respect to the direction of the beam propagation. In these cases it is necessary to take into account projecting the element of area of a reference surface perpendicular to direction of beam propagation when applying Eq. (5). The total energy of the beam must also be conserved as the beam propagates through the system.

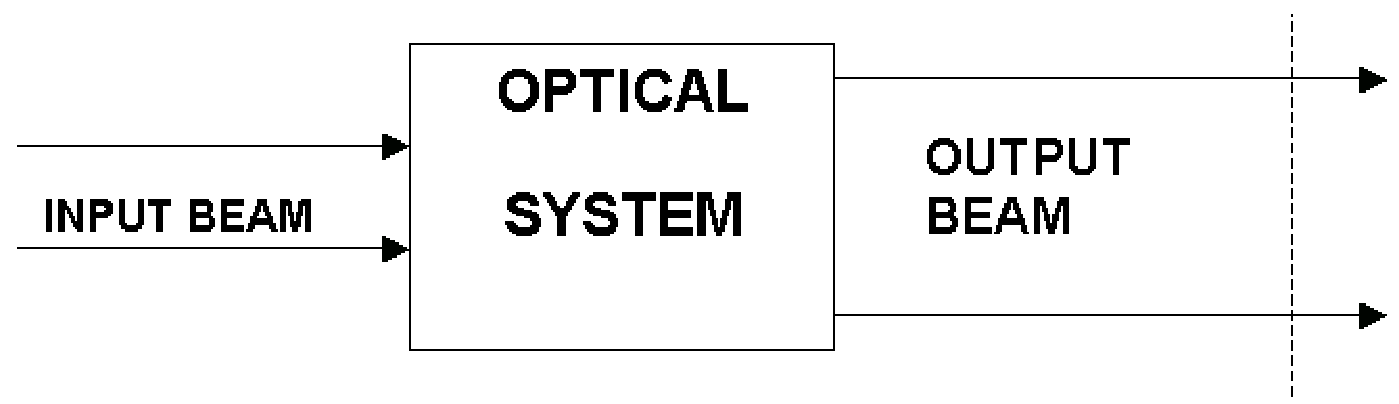

Figure 1. Schematic layout of a laser beam profile shaping system.

Ray tracing ${ }^{48}$ is widely used to simulate the performance of both imaging and non-imaging optical systems. By assigning each incoming ray equal energy density, then by counting the number of rays crossing a unit of area within the optical system, the irradiance can be computed throughout the optical system. Kock ${ }^{45}$ reports a method to simplify photo-radiometric calculations of optical systems by using a reference sphere and ray tracing. The flux flow equation ${ }^{21}$, $46,49,50$ offers an alternate approach for evaluating the irradiance within an optical system. The flux flow equation along with the ray trace equations are used to monitor the change in size of an element of area of a bundle of rays ${ }^{47}$ as the wavefront propagates through the optical system. The flux flow equation depends on the beam parameters and the shape/orientation of the optical surfaces and allows the irradiance to be computed along a ray path as it propagates 
through an optical system. The flux flow equation can also be considered as a differential equation of the optical surface contour, which can be solved if the input and output beam profiles are known.

In order to accomplish the overall beam expansion and profile shaping as illustrated in Figure 1, two optical elements are required. These optical elements may be either lenses or mirrors. The first optical element typically expands and shapes the beam profile to satisfy conservation of energy according to Eq. (5), and the second optical element re-collimates the rays so that the constant optical path length condition is satisfied by the system, as illustrated by the two-lens beam shaping system shown in Figure 2. Optical design of a laser beam shaping system seeks to define the optical components adequately so that the system can be analyzed, fabricated, and tested. This generally requires specification of the shape and spacing between the optical surfaces as well as the index of refraction of all the media.

\subsection{Rotationally Symmetric Systems}

When the incident beam enters the system at a distance $\mathrm{r}$ from the optical axis with an intensity distribution $I_{\text {in }}(r)$, this beam leaves the optical system at a radial distance R from the optical axis with a power density of $I_{\text {out }}(\mathrm{R})$. Integrating Eq. (5) over the input and output planes gives

$$
\int_{0}^{2 \pi} d \theta \int_{0}^{r_{\max }} I_{i n}(r) r d r=\int_{0}^{2 \pi} d \theta \int_{0}^{R_{\max }} I_{\text {out }}(R) R d R .
$$

When $I_{\text {out }}(R)$ is constant, the right hand side of Eq. (6) integrates to give the total power, $\pi I_{\text {out }} R^{2}{ }_{\text {max }}$, passing through the system. One of the two parameters, $R_{\text {out }}$ or $I_{\text {out }}$, may be arbitrarily specified. The other is determined from Eq. (6) to give

$$
R_{\max }=\sqrt{\left(\frac{2}{I_{\text {out }}}\right)^{r_{\max }} \int_{0}^{m_{\text {in }}}(r) r d r}, \quad \text { or } I_{\text {out }}=\left(\frac{2}{R_{\max }^{2}}\right) \int_{0}^{r_{\max }} I_{\text {in }}(r) r d r .
$$

Once $I_{\text {out }}$ is known, the radius of an arbitrary ray in the output beam is given by:

$$
R(r)= \pm \sqrt{\left(\frac{2}{I_{\text {out }}}\right) \int_{0}^{r} I_{\text {in }}(u) u d u}
$$

where $r$ is the radius or distance from the optical axis of the ray in the entrance pupil. The positive sign is used in Eq. (8) when the rays are divergent from the first lens, i.e., the lens surface $s$ is concaved, as shown in Figure 2. The negative sign is used when the rays are convergent from the first lens, i.e., the lens surface s is concaved, and the rays come to a focus and cross the optical axis before reaching the second lens.

In the fundamental, Gaussian $\mathrm{TEM}_{00}$ mode of a laser when the central intensity has been normalized to unity, the input beam profile is given by

$$
I_{i n}(r)=\exp \left[-2(r / r)^{2}\right]
$$

where $r_{0}$ is the beam radius or waist. Alternatively, when $\mathrm{P}$ is the total power of the incident beam, the input intensity profile is given by

$$
I_{\text {in }}(r)=\frac{P \exp \left[-2\left(r / r_{0}\right)^{2}\right]}{\left(\pi r_{0}^{2} / 2\right)\left\{1-\exp \left[-2\left(r_{\max } / r_{0}\right)^{2}\right]\right\}}
$$

where $r_{\max }$ is the radius of input aperture or lens. It is also helpful to note some authors characterize the input Gaussian beam profile in terms of another constant $(\alpha)$ equal to half the beam waist, i.e., $\alpha=r_{0} / 2$. When the input beam profile is given by Eq.(9), the radius of beam in the exit aperture R can be evaluated from Eq. (8) to obtain

$$
R(r)= \pm R_{\max }\left[\frac{1-\exp \left(-2 r^{2} / r_{0}^{2}\right)}{1-\exp \left(-2 r_{\max }^{2} / r_{0}^{2}\right)}\right]^{1 / 2} \text {. }
$$


Equation (11) is used during the optical design process to reduce the number of independent variables when solving for the shape of the reflecting or refracting surfaces of the beam shaping system.

The conservation of energy condition and the constant optical path length condition can be solved simultaneously with the ray trace equations for $R(r), z(r), Z(R)$ when design parameters, such as, $n, d, t_{1}, t_{2}$ for the two-element system shown in Figure 2, are given. It is interesting to note that several authors ${ }^{26,27,40}$ have shown that the sag of two optical elements of a laser beam shaping system can be expressed as a function of $r$

$$
\begin{gathered}
z(r)=\int f(r) d r+C \\
Z(r)=z(r)+g(r)
\end{gathered}
$$

where $\mathrm{C}$ is a constant, and $f(\mathrm{r})$ and $\mathrm{g}(\mathrm{r})$ are functions defining the optical configurations.

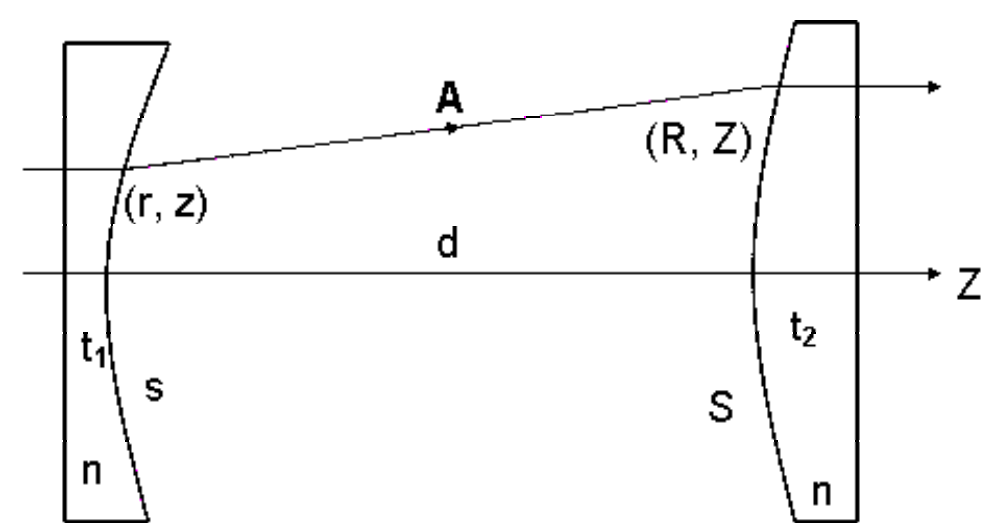

Figure 2. Geometrical configuration of a two-lens laser expander. (From Ref. 33.)

\subsubsection{One-mirror beam shaping system}

Consider the rotationally symmetric geometry of a one-mirror beam shaping system shown in Figure 3 . The radiation is incident upon the mirror surface $\mathrm{s}$ defined by $\mathrm{z}=\mathrm{z}(\mathrm{r})$. The equation of the receiver (detector) surface $\mathrm{S}$ is given by $\mathrm{Z}=$ $\mathrm{Z}(\mathrm{R})$. The irradiance of the incident beam, $I_{i n}(r)$, is incident upon a circular ring about the z-axis of area $\mathrm{da}=2 \pi \mathrm{rdr}$ and is reflected to a circular ring on the receiver surface $S$ of area $d A=2 \pi R\left[1+(d Z / d R)^{2}\right]^{1 / 2}$. The input radiation is collimated (parallel to optical axis) with a known intensity profile. It is desired to irradiate the receiving surface (detector) with a prescribed intensity distribution without placing any conditions on the shape of the wavefront of the output beam. The ray trace equation connecting the mirror surface $\mathrm{s}$ with the receiving surface $\mathrm{S}$ in the $\mathrm{r}-\mathrm{z}$ plane is given by

which can be rewritten as

$$
\frac{R-r}{Z(R)-z(r)}=\frac{\mathbf{A}_{r}}{\mathbf{A}_{z}}=\frac{2 z^{\prime}(r)}{-\left[1-z^{\prime 2}(r)\right]}
$$

$$
-(R-r) z^{\prime 2}+2(Z-z) z^{\prime}+(R-r)=0 \text {. }
$$

Applying the differential energy balance Eq. (5) to this problem gives

$$
I_{\text {in }}(r) 2 \pi r d r=I_{\text {out }}(R) 2 \pi R\left[d R^{2}+d Z^{2}\right]^{1 / 2}=I_{\text {out }}(R) 2 \pi R \sqrt{1+(d Z / d R)^{2}} d R .
$$

where the term $(\mathrm{dR} / \mathrm{dr})$ can be displayed as will be needed in Eq. (17) below. 


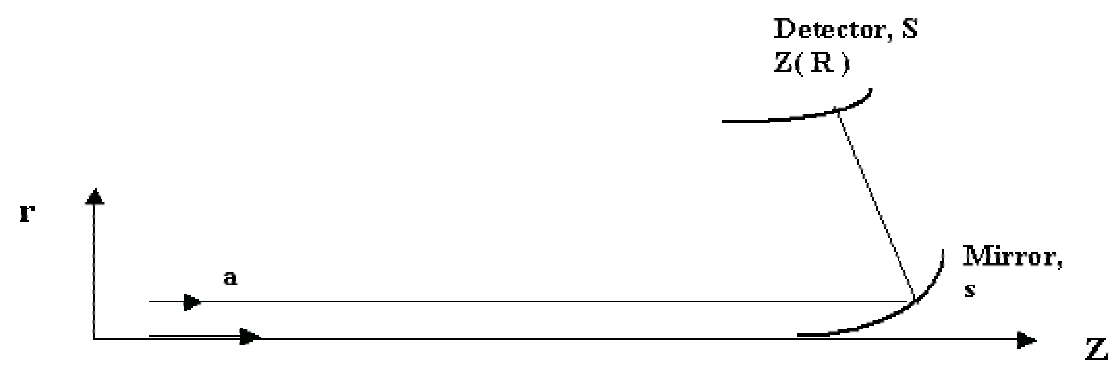

Figure 3. Geometrical configuration of a one-mirror beam profile shaping system.

Recall that $I_{\text {in }}(r), I_{\text {out }}(R)$, and $\mathrm{Z}(\mathrm{R})$ are known functions of their respective variables, and $\mathrm{z}(\mathrm{r})$ is an unknown function at this point of the analysis. Also, note that the ray trace Eq. (14) expresses a mapping between surfaces $\mathrm{S}$ and $\mathrm{S}$, which implies that $\mathrm{R}$ is a function of $\mathrm{r}$. By implicit differentiation of Eq. (15) with respect to $\mathrm{r}$, the differential energy balance equation (16) can be incorporated into the ray trace equation to give:

$$
\frac{z^{\prime \prime}}{z^{\prime}}(R-r)\left[-\left(1+z^{\prime 2}\right)\right]+\frac{d R}{d r}\left[1-z^{\prime 2}+2 z^{\prime} Z^{\prime}\right]-\left(1+z^{\prime 2}\right)=0
$$

where the chain rule for differentiation of the function of a function term $(\mathrm{dZ} / \mathrm{dr})$ and Eq. (15) have been used. The term $(\mathrm{dR} / \mathrm{dr})$ can be eliminated between Eqs. (16) and (17) to give the following differential equation which determines the sag z (r) of mirror surface s:

$$
\frac{z^{\prime \prime}}{z^{\prime}}=\frac{1}{(R-r)}\left\{\frac{I_{\text {in }}(r)}{I_{\text {out }}(R)}\left(\frac{r}{R}\right) \frac{\left[\left(\frac{1-z^{\prime 2}}{1+z^{\prime 2}}\right)+\frac{2 z^{\prime}}{\left(1+z^{\prime 2}\right)}\left(\frac{d Z}{d R}\right)\right]}{\sqrt{1+\left(\frac{d Z}{d R}\right)^{2}}}-1\right\} .
$$

Equation (18) is equivalent to Eq. 3.14 of Ref. 12 or Eq. 13 of Ref. 13. When appropriate boundary conditions are given, Eq. (18) can be solved for the shape of the mirror used to illuminate the receiver surface $\mathrm{S}$ with a prescribed intensity $I_{\text {out }}(R)$ for a given source intensity profile $I_{\text {in }}(r)$. References 12 and 13 develop an extension of this analysis to two-mirror intensity profile shaping systems. A number of specific solutions for both one- and two-mirror systems are given in Refs. 12 and 13 including two laser beam profile shaping systems, such as, uniform illumination of a plane perpendicular to the incident beam using a one-mirror system for an input Gaussian beam, Fig. 7.3 of Ref. 12, and with a two-mirror system, Fig. 7.9 of Ref. 12.

\subsubsection{Optical design of two-lens beam shaping system}

A summary of the historical development of the optical design of a two-lens laser beam shaping system with rotational symmetry is summarized in this section. By shaping two optical surfaces, it is possible to expand and shape the beam profile to satisfy the geometrical optics intensity law and also re-collimate the rays passing through the system so that the constant optical path length condition is satisfied.

\section{Optical design method used by Frieden}

Frieden ${ }^{28}$ describes how to design an optical system for converting the plane wave from the fundamental Gaussian mode of a laser to a plane wave with uniform irradiance over a required cross section with all the power of the incident beam. Frieden assumes that the irradiance of the input beam is given by Eq. (10), expressed in terms of $r_{0}=2 \alpha$. Then, in a plane B a known distance from input plane A, the irradiance is uniform. Using geometrical optics, it follows that light 
within any ring of radii $r, r+\Delta r$ on plane A will strike plane $B$ within another ring of radii $R, R+\Delta R$ where the path for each ray height $\mathrm{R}$ ( $\mathrm{r}$ ) follows from the geometrical optics law of intensity or Eq. (8) which is in this case

$$
R(r)= \pm R_{\max }\left[\frac{1-\exp \left(-r^{2} / 2 \alpha^{2}\right)}{1-\exp \left(-r_{\max }^{2} / 2 \alpha^{2}\right)}\right]^{1 / 2} .
$$

The irradiance is uniform over the plane $\mathrm{B}$, but the radiation is not a plane wave at $\mathrm{B}$, since $\mathrm{R}(\mathrm{r})$ is nonlinear function of $r$. Therefore, a second optical element is required to achieve a plane wave over the plane B.

An expanded view of the two plano-aspheric lenses used for beam shaping is shown in Figure 4. Frieden notes

$$
\tan \theta=\frac{R(r)-r}{Z(R)-z(r)} \cong \frac{R(r)-r}{t-z(r)}
$$

where the approximation $[Z(R) \approx t]$ has been made in writing the right-hand side of Eq. (20). The slope of the surface $s$ is given by

$$
\tan \theta_{1}=\frac{d z(r)}{d r} \equiv z^{\prime}(r) .
$$

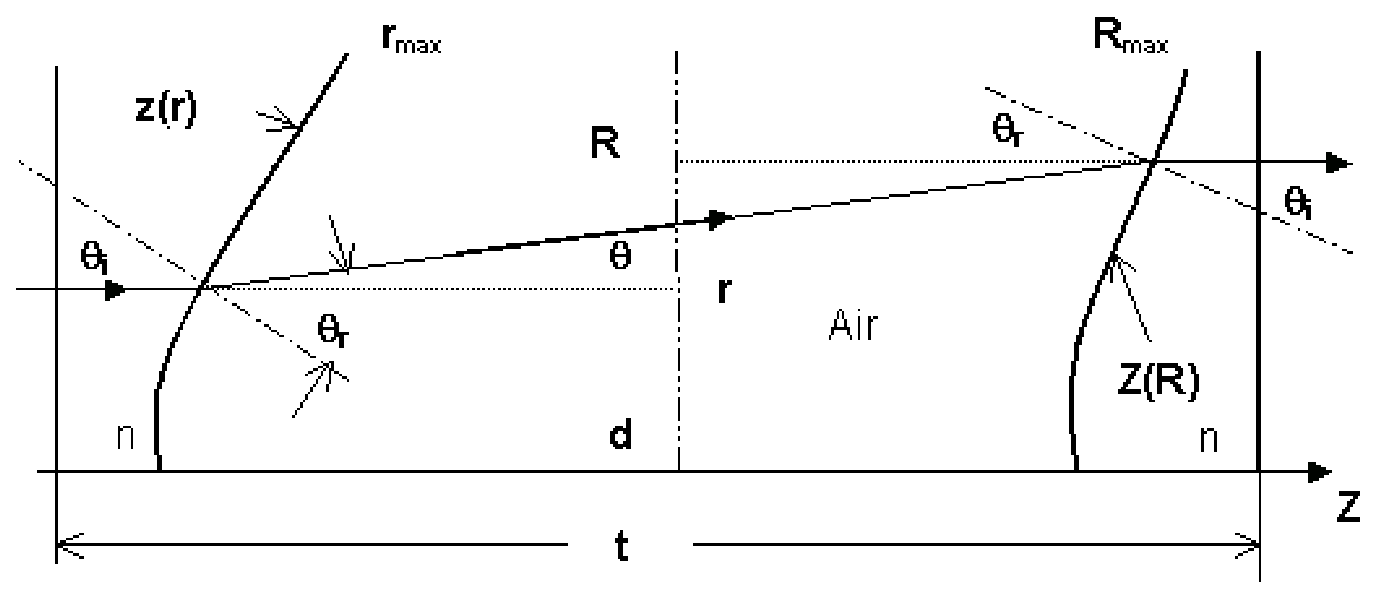

Figure 4. Expanded view of geometrical configuration of laser beam shaping system.

Applying Snell's law to rays refracted at $\mathrm{z}(\mathrm{r})$ expressed in terms of $\tan \theta_{\mathrm{r}}$ gives

$$
\tan \theta_{r}=n z^{\prime} / \sqrt{1-\left(n^{2}-1\right) z^{\prime 2}} .
$$

Using trigonometric identity for $\tan \left(\theta_{\mathrm{r}}-\theta_{1}\right)=\tan \theta$ with Eqs. (21) and (22) allows Eq. (20) to be written as a nonlinear, first-order differential equation for $\mathrm{z}(\mathrm{r})$, the sag of surface $\mathrm{s}$

$$
\frac{n z^{\prime}-z^{\prime} \sqrt{1-\left(n^{2}-1\right) z^{\prime 2}}}{n z^{\prime 2}+\sqrt{1-\left(n^{2}-1\right) z^{\prime 2}}}=\tan \theta=\frac{R(r)-r}{Z(R)-z(r)} \cong \frac{R(r)-r}{t-z(r)} .
$$

Equation (23) can be solved numerically using Eq. (19) for the shape of the aspheric lens surface s, which will uniformly illuminate the plane B. Frieden presents an approximate solution of Eq. (23) by assuming $t>>$ such that $(t-z) \approx t$ and $1-\left(\mathrm{n}^{2}-1\right) \mathrm{z}^{\prime 2} \approx 1$. Then, Eq. (23) can be written as

$$
\frac{(n-1) z^{\prime}}{1+n z^{\prime 2}}=\frac{R(r)-r}{t},
$$

which can be written as a quadratic equation in $z^{\prime}$ 


$$
n(R-r) z^{\prime 2}-t(n-1) z^{\prime 2}+(R-r)=0
$$

Equation (25) has the following solutions

$$
z^{\prime}=\frac{t(n-1) \pm \sqrt{(n-1)^{2} t^{2}-4 n(R-r)^{2}}}{2 n(R-r)} .
$$

Assuming $(\mathrm{n}-1) \mathrm{t}>2 \mathrm{n}(\mathrm{R}-\mathrm{r})$, the square root in Eq. (26) can be expanded to yield

$$
z^{\prime}=\frac{t(n-1) \pm t(n-1)\left[1-\frac{2 n(R-r)^{2}}{(n-1)^{2} t^{2}}+\ldots\right]}{2 n(R-r)} \cong \frac{(R-r)}{t(n-1)}
$$

where the negative sign is Eq. (27) was used to give a positive slope of the surface s. Integrating Eq. (27) with Eq. (19) for $\mathrm{R}(\mathrm{r})$ gives the following expression for $\mathrm{z}(\mathrm{r})$ :

$$
z(r)=\frac{R_{\max }}{t(n-1) \sqrt{1-\exp \left(-r_{0}^{2} / 2 \alpha^{2}\right)}} \int_{0}^{r} \sqrt{1-\exp \left(-u^{2} / 2 \alpha^{2}\right)} d u-\frac{r^{2}}{2 t(n-1)}+t_{1} .
$$

Frieden uses Simpson's rule to compute numerical values for the sag of surface $\mathrm{s}$ for a beam shaping optics with the following parameters: $r_{0}=R_{\max }=2 \mathrm{~mm} ; \mathrm{t}=20 \mathrm{~mm} ; \mathrm{t}_{1}=\mathrm{t}_{2}=5 \mathrm{~mm}, \alpha=1.4 \mathrm{~mm}$, and $\mathrm{n}=1.5$. Frieden notes that there is maximum optical path length variations over the output pupil plane of this system of $20 \lambda$. Therefore, for most laser applications, a second beam shaping optic must be used to re-collimate rays over the output pupil.

Frieden seeks to determine a functional relationship for the sag $\mathrm{Z}(\mathrm{R})$ of surface $\mathrm{S}$ such that any ray passing through the system leave parallel to the optical axis. Applying Snell's law at surface $\mathrm{S}$ gives

$$
\sin \Theta_{r}=n^{\prime} \sin \Theta_{1}=n^{\prime} Z^{\prime} / \sqrt{1+Z^{\prime 2}} .
$$

where this form of Snell's law assumes the output beam is parallel to the optical axis. Noting that $\Theta_{\mathrm{r}}=\theta+\Theta_{1}$ permits writing the following expression

$$
\begin{aligned}
\sin \Theta_{r} & =\sin \left(\theta+\Theta_{1}\right)=\sin \theta \cos \Theta_{1}+\cos \theta \sin \Theta_{1} \\
& =\left[\frac{n z^{\prime}-z^{\prime} \sqrt{1+\left(1-n^{2}\right) z^{\prime 2}}}{1+z^{\prime 2}}\right]\left[\frac{1}{\sqrt{1+Z^{\prime 2}}}\right]+\left[\frac{n z^{\prime 2}+\sqrt{1+\left(1-n^{2}\right) z^{\prime 2}}}{1+z^{\prime 2}}\right]\left[\frac{Z^{\prime}}{\sqrt{1+Z^{\prime 2}}}\right] .
\end{aligned}
$$

Combining Eqs. (23), (29), and (30) lead to the following results

$$
n z^{\prime}-z^{\prime} \sqrt{1+\left(1-n^{2}\right) z^{\prime 2}}+Z^{\prime}\left\{n z^{\prime 2}+\sqrt{1+\left(1-n^{2}\right) z^{\prime 2}}\right\}=n^{\prime} Z^{\prime}\left(1+z^{\prime 2}\right) .
$$

which is consistent with Eq. (12) of Ref.30. It is interesting to note that when $n=n^{\prime}$, then $Z^{\prime}=z^{\prime}$ is a solution to Eq. (31) as reported in the literature. ${ }^{30,31}$ Frieden presents an quadratic equation for $Z^{\prime}$ and a numerical algorithm for computing $Z(R)$ so that optical path difference over the entire pupil is now less than $\lambda / 20$. It is interesting to note that careful analysis of Figure 4 leads to the conclusion that when both lenses have the same index of refraction and when the input and output rays are parallel to the optical axis, then the slope of the two aspherical surfaces must be equal along a given ray. Therefore, further analysis of Eq. (31) does not seem to relate to practical laser beam shaping systems.

\section{Optical design method used by Kruezer}

Kreuzer $^{29}$ also developed a geometrical optics-based method to redistribute the rays of an input laser beam to yield an output beam with a prescribed intensity distribution while maintaining constant optical path lengths between the input and output wavefronts. Kreuzer has presented design equations for a two-element refractive system that will transform a collimated input Gaussian beam into a collimated output beam with uniform irradiance as illustrated in Figure 2. Equation (9) gives the intensity of an input beam in Gaussian $\mathrm{TEM}_{00}$ mode with central intensity normalized to unity. Rays entering the system at a radial distance $\mathrm{r}$ leave the system with an output intensity of $I_{\text {out }}(R)$ at a radial distance $\mathrm{R}$ 
as given by Eq. (11) where the positive solution of $\mathrm{R}$ is used for the configuration considered in Figure 2. Based on the geometrical configuration shown in Figure 4,

$$
R-r=\mathfrak{R}(s, S) \sin \theta .
$$

where $\mathfrak{R}=\sqrt{(R-r)^{2}+(Z-z)^{2}}$. Eliminating $R$ between Eqs. (11) and (32) gives

$$
r+\mathfrak{R}(s, S) \sin \theta-R_{\max }\left[\frac{1-e^{-2\left(r / r_{0}\right)^{2}}}{1-e^{-2\left(r_{\max } / r_{0}\right)^{2}}}\right]^{1 / 2}=0
$$

which must be satisfied by values of $r$ on the refracting surface $\mathrm{s}$ in order to redistribute the input beam profile according to the requirement of conservation of energy.

When both the input and output wavefronts are planar, all rays passing through the beam shaping system must have the same optical path length. Equating the optical path length of an axial and non-axial ray leads to

$$
d(n-1)+\mathfrak{R}(1-n \cos \theta)=0 .
$$

Snell's law for rays refracted at surface $\mathrm{s}$ towards $\mathrm{S}$ is given by

$$
n \sin \theta_{1}=\sin \theta_{r}=\sin \left(\theta+\theta_{1}\right)=\sin \theta_{1} \cos \theta+\sin \theta \cos \theta_{1} .
$$

From Figure $4, \tan \theta_{1}$ is the slope of surfaces $\mathrm{S}$ and $\mathrm{S}$, or

$$
\tan \theta_{1}=\frac{d z(r)}{d r}=\frac{d Z(R)}{d R}
$$

A goal of this analysis is to express $\mathrm{z}(\mathrm{r})$ and $\mathrm{Z}(\mathrm{R})$ in terms of $\mathrm{n}, \mathrm{d}$, and $\mathrm{r}$. Kreuzer proceeds by eliminating $\mathfrak{R}$ between Eqs. (32) and (34) to give

$$
\frac{(1-n \cos \theta)}{\sin \theta}=-\frac{(n-1) d}{(R-r)} .
$$

Dividing Eq. (35) by $\cos \theta_{1}$ and displaying $\tan \theta_{1}$ gives

$$
\tan \theta_{1}=\frac{\sin \theta}{(\cos \theta-n)}
$$

Eliminating $\theta$ between Eqs. (37) and (38) will give a first order differential equation for $\mathrm{z}(\mathrm{r})$. Kreuzer obtains this result by squaring Eq. (37) and adding to $\left(\mathrm{n}^{2}-1\right)$

$$
\left(\frac{(n-1) d}{(R-r)}\right)^{2}+\left(n^{2}-1\right)=\frac{\left(1-2 n \cos \theta+n^{2} \cos ^{2} \theta\right)}{\sin ^{2} \theta}+\left(n^{2}-1\right)=\frac{\left(\cos ^{2} \theta-2 n \cos \theta+n^{2}\right)}{\sin ^{2} \theta}
$$

Noting that the right-hand side of Eq. (39) is the square of the inverse of the right-hand side of Eq. (38) allows one to write the following expression for the slope of surfaces $\mathrm{s}$ and $\mathrm{S}$

$$
\tan \theta_{1}=\frac{1}{\sqrt{\left(n^{2}-1\right)+\left(\frac{(n-1) d}{(R-r)}\right)^{2}}}
$$

Integrating the first Eq. (36) with Eq. (40) leads to an expression for $\mathrm{z}(\mathrm{r})$ which defines $\mathrm{s}$

$$
z(r)=\int_{0}^{r} \frac{d r}{\sqrt{\left(n^{2}-1\right)+\left(\frac{(n-1) d}{(R-r)}\right)^{2}}}
$$

where Eq. (11) expresses R (r). Similarly, integrating the second Eq. (36) with Eq. (40) leads to an expression for Z(R) which defines $\mathrm{S}$ 


$$
Z(R)=\int_{0}^{R} \frac{d R}{\sqrt{\left(n^{2}-1\right)+\left(\frac{(n-1) d}{(R-r)}\right)^{2}}}
$$

where inverting Eq. (11) expresses

$$
r(R)=r_{0} \sqrt{-\frac{1}{2} \ln \left\{1-\left(\frac{R}{R_{\max }}\right)^{2}\left[1-\mathrm{e}^{\left.-2\left(\frac{r_{\max }}{r_{0}}\right)^{2}\right]}\right\}\right.}
$$

In summary, the first lens is a plano-aspheric element where the sag of the aspheric surface is defined by Eq. (41) using Eq. (11) for R (r). The second lens is an aspheric-plano element where the sag of the aspheric is defined by Eq. (42) where $\mathrm{r}(\mathrm{R})$ is given by Eq. (43). Kreuzer notes that if $d$ is sufficiently large, then the term $\left(\mathrm{n}^{2}-1\right)$ in Eqs. (41) and (42) can be neglected. Then, one has

$$
z(r) \cong \frac{1}{(n-1) d} \int_{0}^{r}(R-r) d r .
$$

Kreuzer also presents an example solution for the shape of the aspherical surfaces by tabulating $\mathrm{z}(\mathrm{r})$ and $\mathrm{Z}(\mathrm{R})$ for $\mathrm{n}=$ $1.5117, \mathrm{~d}=150 \mathrm{~mm}, \mathrm{r}_{\max }=\mathrm{R}_{\max }=15 \mathrm{~mm}$, and $\mathrm{r}_{0}=14 \mathrm{~mm}$. In this case, ninety-percent of the input beam is incident upon the beam shaping optics. Kreuzer further notes that the first lens can be a focusing, convex-aspheric element whose sag can be evaluated by replacing $\mathrm{R}$ by $-\mathrm{R}$ in the above equations.

\section{Optical design method used by Rhodes, Jiang, and Shealy}

Both the optical design methods of Frieden and Kreuzer involve solving couple differential equations or two integral equations for the sag of the two refracting surfaces. Rhodes, Jiang, and Shealy ${ }^{30,33}$ simplify the optical design process by showing that the sag of two aspheric surfaces satisfy equations in the form of Eqs. (12) and (13). For more details and application of these results, see Refs. 31,34, 35, 40, and 41. Consider the configuration of a refracting laser beam shaping system shown in Figure 2. The two curved surfaces are used to satisfy the laser beam shaping design conditions. Rays are refracted at surface s according to Snell's law. The ray trace equation of refracted ray A traveling from the point $(\mathrm{r}, \mathrm{z})$ on surface $\mathrm{s}$ to the point $(\mathrm{R}, \mathrm{Z})$ on surface $\mathrm{S}$ is given by

$$
\frac{(\mathrm{R}-\mathrm{r})}{(Z-z)}=\frac{\mathbf{A}_{r}}{\mathbf{A}_{z}}=\frac{z^{\prime}\left[n-\sqrt{1+z^{\prime 2}\left(1-n^{2}\right)}\right]}{n z^{\prime 2}+\sqrt{1+z^{\prime 2}\left(1-n^{2}\right)}} .
$$

Equation (45) can be expressed as a quadratic equation in $z^{\prime}$ and solved to yield

$$
z^{\prime}=\frac{-(R-r)(Z-z) \pm n \sqrt{(Z-z)^{2}+(R-r)^{2}}}{\left[1-n^{2}\right](Z-z)^{2}-n^{2}(R-r)^{2}} .
$$

The positive solution for $z^{\prime}$ is used for the lens configuration shown in Figure 2 where the first lens is divergent. For this system, the height of the ray $R$ at the second lens with entrance pupil height $r$ is computed Eq. (11) with the positive solution. The term $(Z-z)$ in Eq. (46) is determined by the constant optical path length condition realized by setting the axial optical path length equal to that of a general ray

$$
\left[(R-r)^{2}+(Z-z)^{2}\right]^{1 / 2}=n(Z-z)-d(n-1)
$$

which is a quadratic equation for the term $(Z-z)$ as a function of the entrance pupil aperture radius $r$. After squaring Eq. (47) and collecting terms, the solution of the resulting quadratic equation is 


$$
(Z-z)=\frac{n(n-1) d+\left[(n-1)^{2} d^{2}+\left(n^{2}-1\right)(R-r)^{2}\right]^{1 / 2}}{n^{2}-1}
$$

where the positive sign of the radical has been used so that the solution reduces to the appropriate value of $(Z-z)=d$ when $r=R=0$. It is interesting to note that Eq. (46) permits $z^{\prime}$ to be expressed as a function of $r$, thus, enabling $z$ (r) to be evaluated by integration, as illustrated in Eq. (12). References 33 and 34 present results for design, fabrication, and testing of a two-lens laser beam shaping system similar to configuration shown in Figure 2.

\subsubsection{Analysis, fabrication, and testing of two lens beam shaping systems}

Several optical design methods for calculating the sag of the two aspherical lens surfaces required to shape an input Gaussian beam have been discussed in the previous section. The design methods of Rhodes, Jiang, and Shealy have been used to develop a prototype laser beam shaping system, which has been designed, fabricated and tested. ${ }^{33}$ The lens thickness is not involved in the design differential equations, but is an important factor to be considered, since it relates to the energy absorption by the optics. The lens thickness of each element has been assumed to be $10 \mathrm{~mm}$ for this system. Another system parameter, the distance between the two lenses is important for optical design, fabrication, and testing of the system. The larger the spacing between the two elements, the smaller the surface curvature of each element required to satisfy the design conditions. However, if the element spacing is too large, the system will be difficult to assemble and test. The distance between these elements has been arbitrarily chosen for this system to be five times the diameter of the lens elements.

Table 1. Surface parameters of a $\mathrm{HeCd}(441.57 \mathrm{~nm})$ laser profile shaping system where the distance between the primary and secondary lens is $150 \mathrm{~mm}$. (From Ref. 40 )

\begin{tabular}{|c|c|c|}
\hline Lens Surface Parameters & Primary Mirror & SecondaryMirror \\
\hline Diameter $(\mathrm{mm})$ & 30.0 & 30.0 \\
\hline Vertex Radius $(\mathrm{mm})$ & 47.861445 & 113.64905 \\
\hline Index of Refraction & $1.43916\left(\mathrm{CaF}_{2}\right)$ & $1.43916\left(\mathrm{CaF}_{2}\right)$ \\
\hline Thickness $(\mathrm{mm})$ & 10.0 & -1.4877144 \\
\hline Conic Constant, $\kappa$ & -1.1143607 & $-2.6522455 \times 10^{-6}$ \\
\hline $\mathrm{A}_{4}\left(\mathrm{~mm}^{-3}\right)$ & $-7.1532887 \times 10^{-5}$ & $9.4058758 \times 10^{-9}$ \\
\hline $\mathrm{A}_{6}\left(\mathrm{~mm}^{-5}\right)$ & $3.3729843 \times 10^{-7}$ & $-2.3096843 \times 10^{-10}$ \\
\hline $\mathrm{A}_{8}\left(\mathrm{~mm}^{-7}\right)$ & $-1.4916816 \times 10^{-9}$ & $1.5839557 \times 10^{-12}$ \\
\hline $\mathrm{A}_{10}\left(\mathrm{~mm}^{-9}\right)$ & $5.9836543 \times 10^{-12}$ & $-4.8438745 \times 10^{-15}$ \\
\hline $\mathrm{A}_{12}\left(\mathrm{~mm}^{-11}\right)$ & $-1.5166511 \times 10^{-14}$ & \\
\hline
\end{tabular}

The index of refraction of the lenses affects the shape of refracting surfaces $\mathrm{s}$ and S. For this application the lens elements must have a high transmission for the wavelength of the laser being used [441.57mm for Helium Cadmium ( $\mathrm{HeCd}$ ) laser] with this prototype laser beam shaping system. There are several materials with good transmission properties at $441.57 \mathrm{~nm}^{51}$ Acrylic (PMMA) Plastic, Calcium Fluoride $\left(\mathrm{CaF}_{2}\right)$, Crown Glass (BK7), Lithium Fluoride (LiF), Fused Quartz, and Fused Silica. Acrylic Plastic is difficult to polish. Fused Quartz, Fused Silica, and Crown Glass are difficult to machine with a single-point diamond lathe as required in making these aspheric surfaces. Lithium Fluoride contains defects and is difficult to work with. Calcium Fluoride has good transmission properties $(\sim 95 \%)$ within the range of $150-9000 \mathrm{~nm}$, can be machined with a single-point diamond lathe, and is also the least expensive of this group of materials. Therefore, $\mathrm{CaF}_{2}$ has been selected as the lens material for this laser beam profile shaping system. The index of refraction of $\mathrm{CaF}_{2}$ is a function ${ }^{52}$ of the wavelength of the light being used:

$$
n^{2}=1+\sum_{i=1}^{3} \frac{A_{i} \lambda^{2}}{\lambda^{2}-\lambda_{i}^{2}}
$$

where $\lambda$ is measured in units of microns $(\mu)$ and $\lambda_{1}=0.05026360 \mu \mathrm{m} ; \lambda_{2}=0.1003909 \mu \mathrm{m} ; \lambda_{3}=34.649040 \mu \mathrm{m} ; \mathrm{A}_{1}=$ 0.5675888; $\mathrm{A}_{2}=0.4710914 ; \mathrm{A}_{1}=3.848723$. Using Eq. (49), the index of refraction of $\mathrm{CaF}_{2}$ has been calculated to be 1.43916 at the $\mathrm{HeCd}$ laser wavelength of $441.57 \mathrm{~nm}$. 
For this laser profile shaping system, the laser beam is expanded from $16 \mathrm{~mm}$ to $25 \mathrm{~mm}$ in diameter while the beam intensity profile is flattened. After solving the differential equations, a non-linear least squares fitting process was used to fit the lens surface data to the conventional optics surface equation

$$
z=\frac{c r^{2}}{1+\sqrt{1-(1+\kappa) c^{2} r^{2}}}+\sum_{i=1}^{N} A_{2 i} r^{2 i}
$$

where $\mathrm{C}$ (vertex curvature), $\kappa$ (conic constant), and $\mathrm{A}_{2 \mathrm{i}}$ (coefficients of the polynomial deformation terms) are surface parameters that are determined by the fitting process for each surface. The data for surfaces $\mathrm{S}$ and $\mathrm{S}$ obtained from solving the differential equations has been fit to many different expressions for the optics surface equation. More aspheric terms will generally give a better fit with smaller least squared errors. However, it is easier to fabricate and test surfaces with a non-zero conic term and a small number of polynomial deformation terms. The data in Table 1 with non-zero conic constant plus five deformation terms represents a compromise between fitting accuracy, optical modeling performance, and ease of fabrication.

\subsubsection{Analysis of Lens Performance}

The optical performance of the optical system defined by Table 1 has been modeled and compared to the design conditions of the laser profile shaping system. Applying the flux flow equation ${ }^{46}$ to this lens system, the intensity of a cross-section of the output beam has been evaluated. A careful computation of the area under the surfaces generated by rotating around the optical axis the input and output intensity profiles given in Figure 7 of Ref. 40 shows that the total power is conserved for this laser beam profile shaping system. The optical path difference (OPD) of the output beam for the shaping system defined in Table 1 has also been evaluated over the aperture. The maximum OPD for this system is $0.0017 \lambda$, which corresponds to the absolute OPD of $0.75 \mathrm{~nm}$ for the HeCd laser. This demonstrates that the shape of the output wavefront has the same shape as the input wavefront, as required by the constant OPL condition
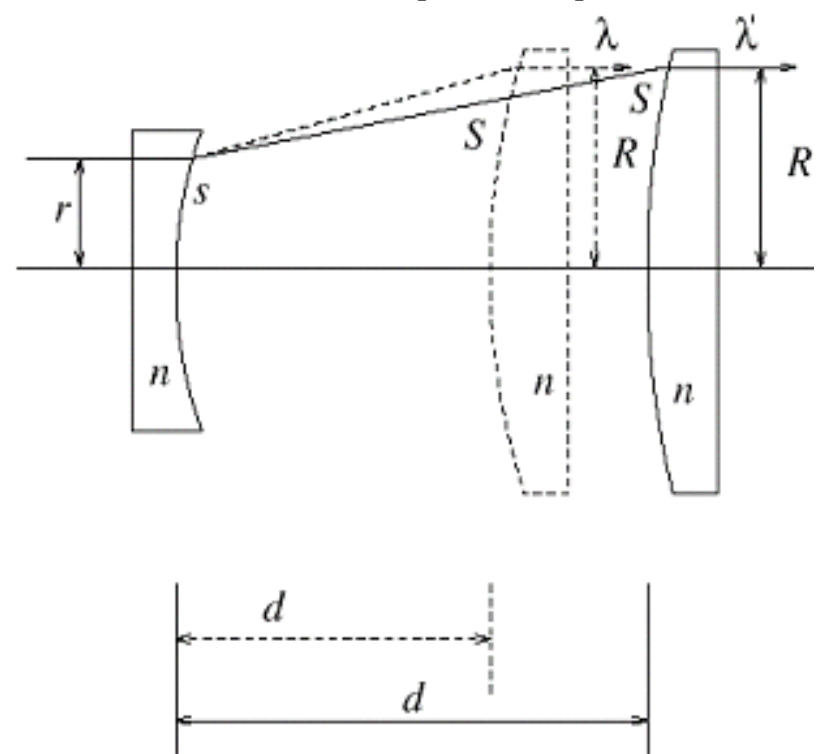

It is also interesting to consider how this laser beam shaping system would perform using a different laser wavelength, $\lambda^{\prime}$. Since the index of refraction is a function of wavelength as given by Eq. (49) and since these optics have been designed for a specific $n(\lambda)$, it is important to determine whether a set of laser beam profile shaping optics can be used for multiple $\lambda$. For example, if the optics defined in Table 1 were used with a Helium Neon ( $\mathrm{HeNe}$ ) laser with wavelength $632.8 \mathrm{~nm}$, the index of refraction of the lenses would be reduced to 1.43289. Then, according to Snell's law, the light leaving the primary lens would not be refracted as strongly as the HeCd light. The separation between the two lenses would need to be increased to accommodate this decrease in index of these lenses to insure that the light is incident upon the secondary at the appropriate height so that it will be refracted parallel to optical axis as illustrated in Figure 5.

Figure 5. Illustrates the relationship $\lambda$ and $d$.

Now, we will derive an expression for the lens separation for a particular ray so that this ray will be incident upon surface $\mathrm{S}$ at a suitable point such that after refraction, this ray will leave the secondary lens at the appropriate height $\mathrm{R}$ to insure conservation of energy and to be parallel to the optical axis. The new distance $d$ can be calculated from Eq. (46), since $r, \mathrm{R}$, and $\mathrm{z}^{\prime}$ are known. Equation (46) can be written as a quadratic equation of $(\mathrm{Z}-\mathrm{z})$ with the physical solution

$$
Z-z=\frac{(R-r)\left[1+n \sqrt{1+\left(1-n^{2}\right) z^{\prime 2}}\right]}{\left(n^{2}-1\right) z^{\prime}} .
$$


Once $(\mathrm{Z}-\mathrm{z})$ is known, then the corresponding value of $d$ can be determined by solving Eq. (48) as a quadratic equation for $\mathrm{d}$ :

$$
\left(n^{2}-1\right)(n-1)^{2} d^{2}-2 n(n-1)\left(n^{2}-1\right)(Z-z) d+\left(n^{2}-1\right)^{2}(Z-z)^{2}-\left(n^{2}-1\right)(R-r)^{2}=0
$$

with the physical solution

$$
d=\frac{n(Z-z)-\sqrt{(Z-z)^{2}+(R-r)^{2}}}{n-1} .
$$

If the lenses are separated by the vertex spacing $d$ of Eq. (53), then the ray leaves the secondary lens parallel to the optical axis. However, every ray in the entrance pupil will require a different lens spacing $\mathrm{d}$. Calculations have shown that the optical system defined in Table 1 will need a lens spacing of approximately $\mathrm{d}=152.18 \mathrm{~mm}$ when used to shape the $632.8 \mathrm{~nm}$ HeNe laser beam profile. The maximum difference between the lens spacing for all rays is less than 10 microns, which is smaller than alignment errors. Therefore, it seems reasonable to use this laser beam profile shaping system at multiple wavelengths.

The two plano-aspherical lenses of Table 1 has fabricated by Janos Technology, Inc. of Townshend, Vermont, using a single point diamond lathe where $\mathrm{CaF}_{2}$ was used as the lens material. A scanning video system was used to measure the input and output beam profiles. A full discussion of these experimental results is presented in Refs. 33 and 34. There was approximately a $10 \%$ variation of the output beam intensity for the system fabricated and made during the early 1990's. The output wavefront shape of this two plano-aspherical lens laser beam profile shaping system has also been evaluated. Imposing the constant optical path length condition requires the output wavefront to have the same geometrical shaping as the input wavefront, which is assumed to be planar. The collimated radiation of the input laser beam has a very small divergence, which means that the beam diameter does not increase or decrease with propagation within limits of geometrical optics. For propagation of the output wavefront of this laser profile shaping system around the laboratory, say $10 \mathrm{~m}$, the output beam diameter remained constant. To quantify this result, Ronchigrams ${ }^{53}$ of the input and output beams were recorded using the video system described in this section for recording the beam intensity profile. ${ }^{54}$ Analysis of these Ronchigrams indicates that the output beam wavefront has the same shape as the input wavefront. In addition, a HeNe laser was used to illuminate these profile shaping optics, and the predictions of section 2.1.4 have also been confirmed. When using a HeNe laser with $632.8 \mathrm{~nm}$ radiation, the lens spacing was increased to $152.2 \mathrm{~mm}$ according to the predictions of Eq. (53).

\subsection{Non-Rotationally Symmetric Systems}

For a projective transformation ${ }^{55}$ in optics, a point in image space can be expressed as a linear function of the coordinates of the object point. Perfect imaging systems, such as, Maxwell's "fish-eye" lens or stigmatic imaging of surfaces, are examples of projective transformations in optics. In practice, aberrations are present in many optical systems, and point-to-point imaging is not possible, except to the first-order or paraxial approximation. Cornwel1 ${ }^{56,57}$ notes that all real optical systems perform non-projective transformations to some extent. That is, there is a non-linear dependence between input (or object) and output (or image) coordinates.

The redistribution of rays leading to the beam shaping allows for transformation of a Gaussian input beam into a more uniform (top-hat, Fermi-Dirac, or super-Gaussian) irradiance output beam requires a non-linear relationship between the input and output aperture coordinates. Therefore, the geometrical methods ${ }^{40}$ for designing a laser beam shaping system are an example of a non-projective transformation in optics. Cornwell notes that the first element of a laser beam profile shaping (non-projective transform) system creates sufficient aberrations in the wavefront to restructure the intensity of the beam after propagation of the wavefront over a specified distance. Then, the second element of a laser beam profile shaping system has suitable contour to restore the original wavefront shape of the beam. If the purpose of a laser beam profile shaping system is to uniformly illuminate a surface, then the second element is not needed. Symbolically, a laser beam profile shaping system may be considered to be a "black box" which transforms an input laser beam (plane wave) with a Gaussian intensity distribution into an output beam (plane wave) with uniform intensity distribution. The input and output beams have radii $r$ and $R$, respectively, as shown in Figure 2. Reference 56 presents extensive discussion of many types of laser beam shaping systems and draws some interesting general conclusions.

In particular, Cornwell provides a seven-step recipe for designing two-element systems, which perform nonprojective transformations, such as, laser profile shaping systems. Since the contents of Ref. 56 are not widely available in the optics literature to the knowledge of this author, these seven steps are summarized below: 
1. Write out differential power expressions for the intensity distributions over the input and output planes.

$\underline{\text { Rectangular Coordinates }}$

$$
I_{\text {in }}(x, y) d x d y=I_{\text {out }}(X, Y) d X d Y
$$

$\underline{\text { Polar Coordinates }}$

$$
I_{\text {in }}(r) r d r=I_{\text {out }}(R) R d R
$$

2. Use the conservation of energy to relate the input and output beam parameters.

$\underline{\text { Rectangular Coordinates }}$

$$
\int_{\text {Input Aperture }} I_{\text {in }}(x, y) d x d y=\int_{\text {Output Aperture }} I_{\text {out }}(X, Y) d X d Y
$$

Polar Coordinates

$$
\int_{\text {Input Aperture }} I_{\text {in }}(r) r d r=\int_{\text {Output Aperture }} I_{\text {out }}(R) R d R
$$

3. Determine the magnification relating the input and output ray heights.

Rectangular Coordinates

Assume the intensity functions are separable

$$
\begin{gathered}
I_{\text {in }}(x, y)=a_{x}(x) a_{y}(y) \\
I_{\text {out }}(X, Y)=A_{X}(X) A_{Y}(Y)
\end{gathered}
$$

Allowing for non-uniform shaping of a laser beam profile in two orthogonal directions, $\mathrm{X}=\mathrm{m}_{\mathrm{x}}(\mathrm{x}) \mathrm{x}$ and $\mathrm{Y}=$ $\mathrm{m}_{\mathrm{y}}(\mathrm{y}) \mathrm{y}$, the rectangular magnifications follow from combining Eqs. (54), (58) and (59)

$$
\begin{aligned}
& m_{x}(x)=\frac{1}{x}\left[C_{1} \int_{0}^{x} \frac{a_{x}(u) d u}{A_{X}\left(u m_{x}(u)\right)}+C_{2}\right] \\
& m_{y}(y)=\frac{1}{y}\left[\frac{1}{C_{1}} \int_{0}^{y} \frac{a_{y}(v) d v}{A_{Y}\left(v m_{y}(v)\right)}+C_{3}\right]
\end{aligned}
$$

where $\mathrm{C}_{\mathrm{i}}$ are constants determined by boundary conditions, such as, the magnification for a rim ray.

Polar Coordinates

$$
\begin{gathered}
R=m(r) r \\
m(r)=\frac{1}{r}\left[2 \int_{0}^{r} \frac{I_{\text {in }}(r) r d r}{I_{\text {out }}(m(r) r)}+C\right]
\end{gathered}
$$

where $\mathrm{C}$ is a constant determined from the boundary conditions.

4. Express the optical path length (OPL) between input and output reference surfaces of an arbitrary ray in terms of the OPL of a reference ray.

5. Determine the $\operatorname{sag} \mathrm{z}(\mathrm{r})$ of the first element.

6. Determine the inverse magnification relating the ray coordinates at the first and second elements.

7. Determine the sag $\mathrm{Z}(\mathrm{R})$ of the second element.

\subsubsection{Optical design of a two-mirror laser beam shaping system}

The seven-step recipe of the previous section has been used to design of a two-mirror laser beam shaping system with rectangular symmetry and no central obscuration. For more details, see Refs. 27 and 41. Figure 6 shows the geometrical configuration of the two-mirror laser beam shaping system considered. The input and output beams are collimated and parallel to the optical axis. Assume the input beam irradiance is given by 


$$
I_{i n}(x, y)=\exp \left[-2\left(\frac{x}{x_{0}}\right)^{2}\right] \exp \left[-2\left(\frac{y}{y_{0}}\right)^{2}\right]
$$

where $\left(\mathrm{x}_{0}, \mathrm{y}_{0}\right)$ are the beam waist in the $\mathrm{x}, \mathrm{y}$ directions, and the central intensity is normalized to unity. The output beam irradiance is uniform. In this case, explicit expressions for $\mathrm{X}(\mathrm{x}), \mathrm{Y}(\mathrm{y}), \mathrm{Z}(\mathrm{x}, \mathrm{y})$, and $\mathrm{Z}(\mathrm{X}, \mathrm{Y})$ have been evaluated. ${ }^{41}$ Using these results, the performance of a two-mirror laser beam shaping system which transforms an input beam with elliptical cross section of 3:1 ratio of beam waist in perpendicular directions has been analyzed. ${ }^{27}$ The aspherical deviation of mirror surfaces from best-fit sphere has been shown to be $120 \mu \mathrm{m}$ for a $6 \mathrm{~mm}$ diameter mirror. The optical analysis software ZEMAX ${ }^{58}$ has been used for performance modeling and tolerancing analysis of this system. These results show that the first mirror surface has a strong aspherical component along the direction of smaller input beam waist and that the output beam profile remains fairly uniform when the mirror decentation is less than $2.5 \%$ of the maximum mirror surface dimension and tiltation is less than 2.5 degrees about the coordinate axis.

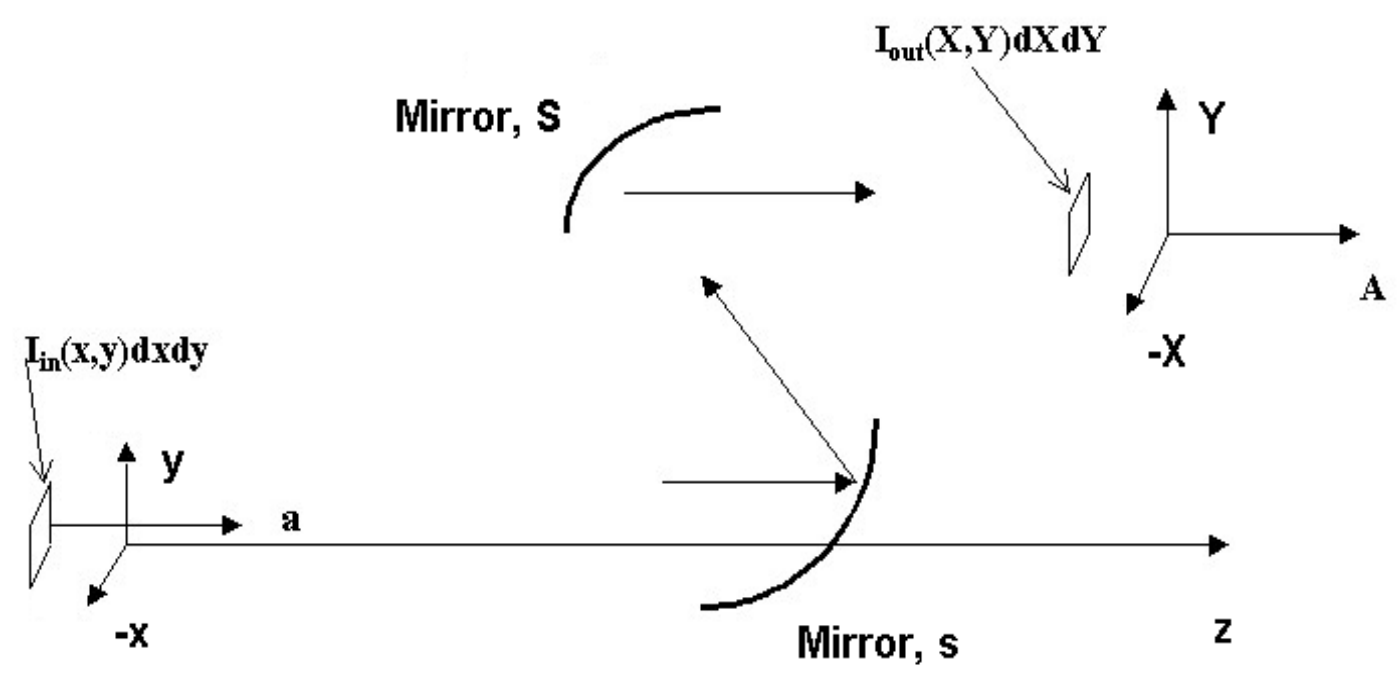

Figure 6. Geometrical configuration of a two-mirror laser beam shaping system with rectangular symmetry. (From Ref. 27)

\subsection{Genetic Algorithm Optimization Method}

For more complex configurations, it is often difficult to obtain analytical solutions to the laser beam shaping design conditions. Genetic algorithm (GA) optimization methods have been shown to work well designing laser beam shaping systems, when the merit function contains both discrete and continuous parameters. ${ }^{42}$ As an example of a more complex problem - one difficult to solve using more conventional methods - the GA technique has been used to design a gradient index (GRIN) laser beam shaping system. Wang and Shealy ${ }^{59}$ solved this problem using a differential-equation design method. Though Wang was able to produce several perfectly good solutions, the GRIN profiles of the lenses reported were determined solely by solving the differential equations, and no constraints were imposed that the GRIN profiles of the lenses correspond to those that can be found in common glass catalogs. It follows that a challenging problem for the GA optimization method would be to create a laser shaping system with spherical-surface GRIN elements, as Wang did, but to do so with the added constraints that catalog GRIN glass types are used and the number of lens element required is an optimization variable. Solution to this problem will represent a way to construct a laser beam shaping system without use of aspherical optics or esoteric GRIN profiles. ${ }^{59}$ The optical design for this system involves using genetic algorithms (GA) to maximize a laser beam shaping merit function, Eq. (65), within a 26-dimensional parameter space. ${ }^{60}$ To solve this optical design problem, the GA not only must optimize surface shapes of the GRIN elements and their spacing, but also must determine the actual number of GRIN elements in the solution, up to a certain limit (four, in this case), and the type of GRIN material for each element must be selected from a vendor GRIN glass catalog. This type of problem distinguishes the GA method from deterministic methods (i.e., those that rely on derivatives and a smooth, continuous 
merit function) since the merit function required for this problem depends on a complicated mix of discrete and continuous parameters. For more details and applications of these results, see Refs. 42, 60, and 61.

The optical design of this system solves for the attributes (radii, thickness, and spacing) of the lens elements, the GRIN glass type from a catalog, and the number of elements needed for a system of this configuration to satisfy the beam shaping design conditions - conservation of energy within beam and constant optical path length condition. Twenty-six parameters of the lens system are determined by the GA optimization. The merit function M includes terms that favor a specific beam diameter, a uniform irradiance output beam profile, and a collimated output beam. The following merit function satisfies these design objectives and is maximized during design:

$$
M=\frac{M_{\text {Diameter }} M_{\text {Collimation }}}{M_{\text {Uniformity }}}=\frac{\exp \left[-s\left(R_{\text {Target }}-R_{\mathrm{N}}\right)^{2}\right] \exp \left[-\left(1-\prod_{i=1}^{N} \cos ^{Q}\left(\gamma_{i}\right)\right)^{2}\right]}{\sqrt{\frac{1}{N} \sum_{i=1}^{N}\left\{I_{\text {out }}\left(R_{i}\right)-\left[\frac{1}{N} \sum_{k=1}^{N} I_{\text {out }}\left(R_{k}\right)\right]\right\}}}
$$

where $R_{\text {Target }}$ is the desired radius of the output beam, $R_{N}$ is the radial height of the marginal ray on the output surface, $\gamma_{i}$ is the angle the $i^{\text {th }}$ ray makes with the optical axis, $s$ and $Q$ are convergence constants used to adjust importance of different components of merit function during optimization, and $\mathrm{I}_{\text {out }}\left(\mathrm{R}_{\mathrm{k}}\right)$ is the irradiance of ray in output beam. (See Ref. 61 for a detailed discussion of this GA optimization process and construction of a suitable merit function to use when designing laser beam shaping systems.) The exponential function is used in the merit function, since it peaks strongly as parameters approach their design targets. Also, as the output beam profile becomes more uniform, the denominator of Eq. (65) approaches zero, and $M$ increases substantially. In summary, the merit function rewards those systems, which tend to increase the value of $M$ and penalize systems with smaller values of $M$ as the GA optimization searches throughout both the discrete and continuous parameter space. After 12,367 generations (iterations), the GA converges to a three-element GRIN lens system with all spherical surfaces which is illustrated in Figure 7. For more discussion of convergence of the GA optimization method for application to laser beam shaping systems, see Ref. 60 .

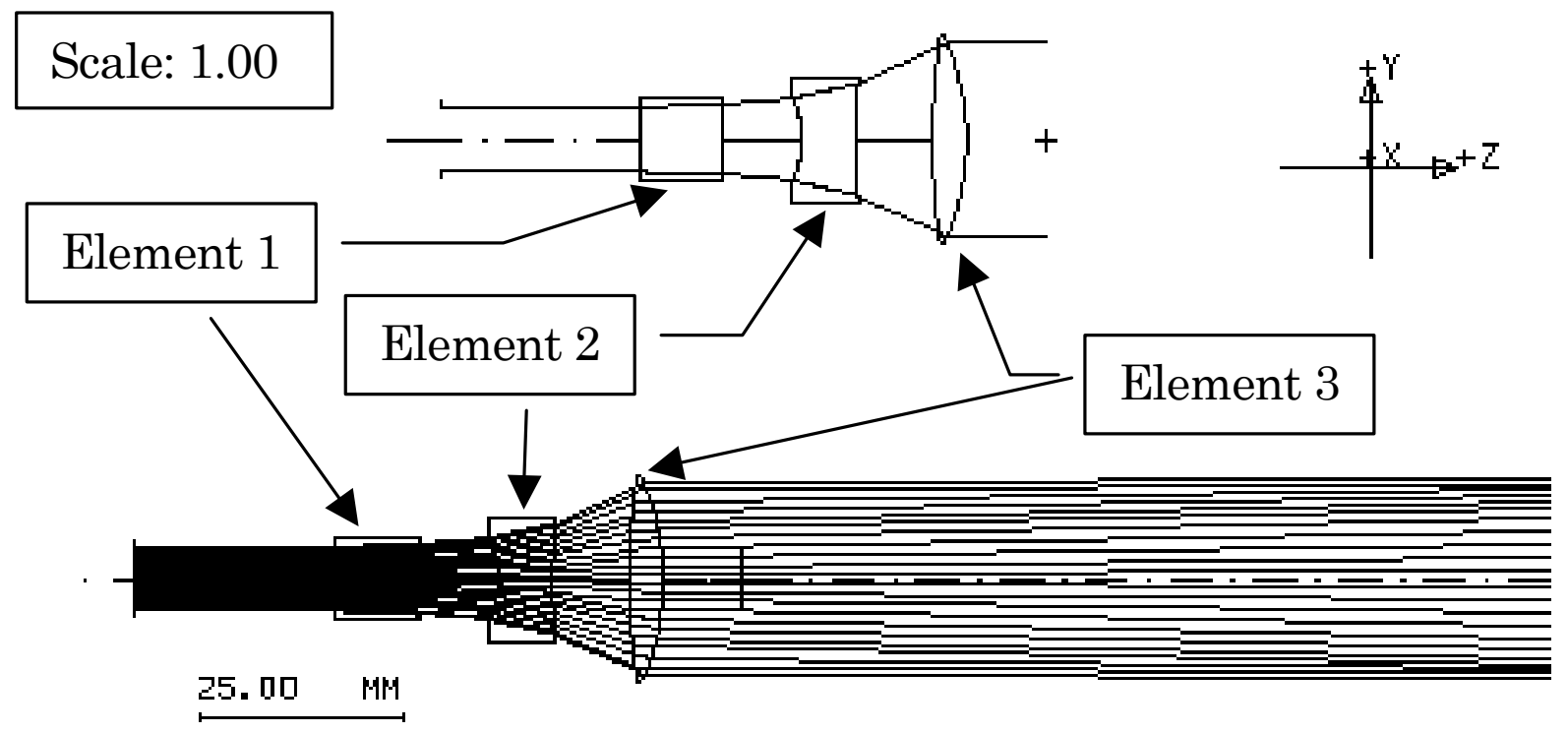

Figure 7. Raytrace for the free-form GA-designed GRIN shaper system. (From Ref. 60)

\section{APPLICATIONS}

Laser beam profile shaping optics are well suited for applications whose overall efficiency increases when the irradiance over the detector (or substrate) is uniform, such as in compact holographic projector systems. ${ }^{62,63,64}$ These compact 
holographic projection systems have been reported to offer a practical way to make a highly corrected mesh or grid pattern over curved surfaces where the pattern can range in size from sub-micron to multi-micron. The laser profile shaping optics within a holographic projection system enables uniform features to be written over substrates of several centimeters in diameter. ${ }^{35}$ To understand this increase in system efficiency when using laser beam shaping optics, note that for a Gaussian beam with irradiance given by Eq. (9), the intensity of the beam decreases to $1 / \mathrm{e}^{2} \approx 13.5 \%$ of its axial value at the beam radius. The effect of this variation in beam intensity over a Gaussian beam is illustrated in Figure 8 where (A) shows significant variation in pattern densities at the center and edge of beam for the same substrate (film) and exposure time when a laser profile shaping optics is not part of the system, and (B) shows almost uniform pattern densities at the center and edge of beam when laser profile shaping optics are part of the system. Therefore, when beam shaping optics are introduced into a holographic projection processing system ${ }^{35}$ as illustrated, the detector substrate will be uniformly illuminated, and photochemical reactions take place at the same rate over the entire substrate area, thus, enabling the full beam diameter to be available for material processing. Introducing laser shaping optics into holographic projection processing systems have lead to a significant increase in quality of micro-optics fabricated over the substrate.
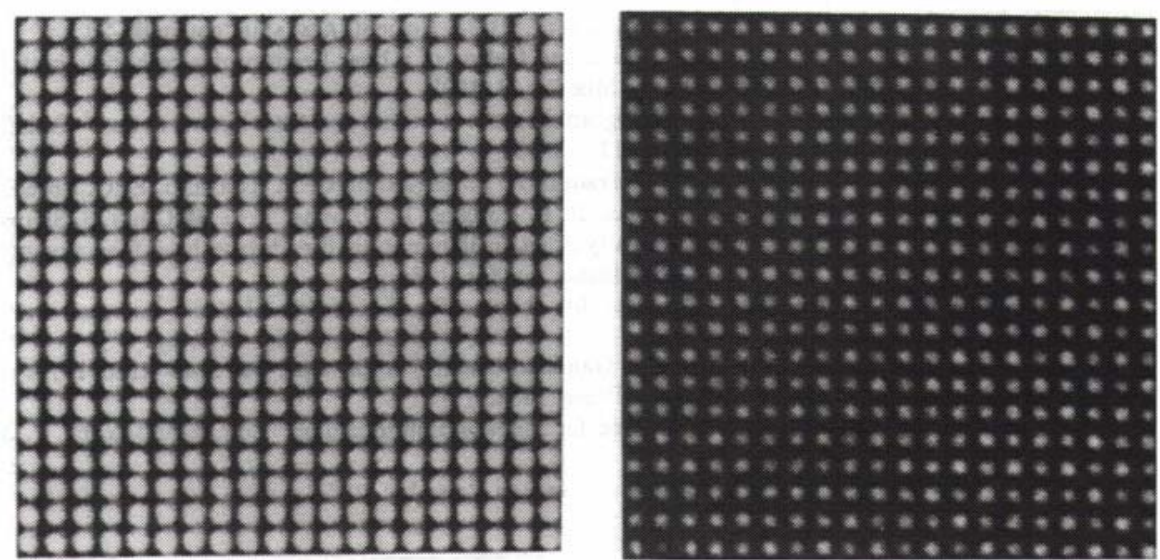

(A)
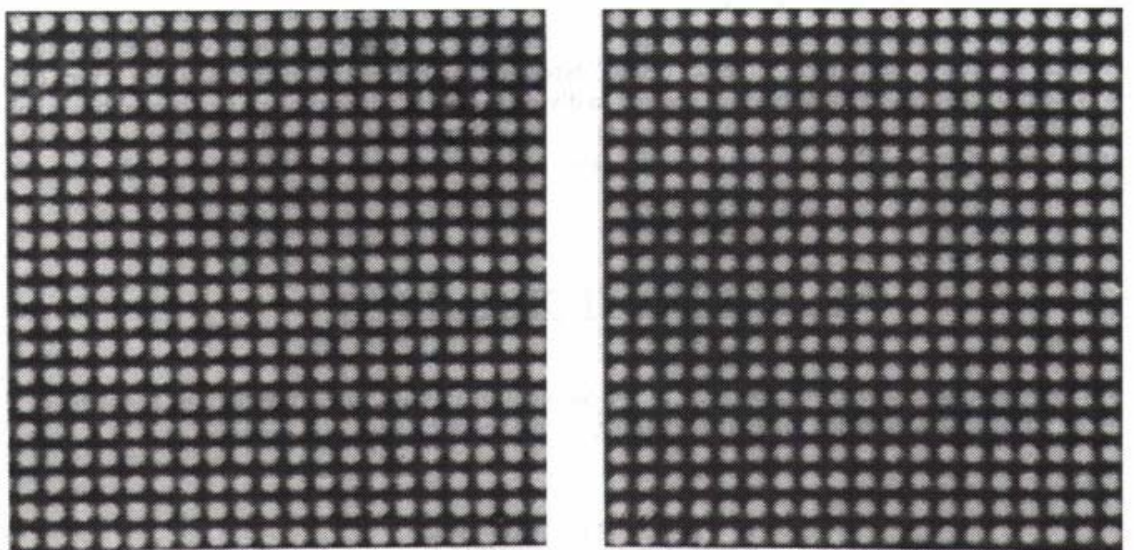

(B)

Figure 8. Interference patterns produced by a 4-beam holographic projection processing system when illuminated with a Gaussian beam. The image on the left side of the figure was taken near the center of the beam, and the image on the right side of the figure was

taken near the edge (waist) of the beam. The images (A) were taken when the laser beam profile optics was not part of projection system, and the images (B) were taken when laser beam profile shaping optics was part of projection system. (From Ref. 34) 


\section{SUMMARY AND CONCLUSIONS}

An overview of the development and application of the geometrical methods for design of laser beam shaping systems has been presented. This geometrical optics-based theory for designing laser beam shaping is based on conservation of radiant energy within a bundle of rays, the ray trace equations, and the constant optical path length condition for cases when contour of the incident wavefront is maintained as the beam passes through the system. This theory has been used to compute the sag of the optical surfaces for two-plano-aspheric lens system and a two-mirror configuration and to setup a laser beam shaping merit function for a genetic algorithm optimization of a three-element GRIN system with spherical surfaces.

In the first example, numerical techniques were used to solve the differential equations for the sag of the shaping elements in a two-lens laser beam shaping system, which has been fabricated and tested. In the second example, analytical and numerical techniques were used to determine and analyze the performance of a two-mirror laser beam shaping system with no central obscuration, which can transform an elliptical Gaussian input beam into a rectangularsymmetric output beam with uniform irradiance. The optical analysis software ZEMAX has been used for performance modeling and tolerance analysis for this system. These results show that the first mirror surface has a strong aspherical component along the direction of smaller input beam waist and that the output beam profile remains fairly uniform when the mirror decentation is less than $2.5 \%$ of the maximum mirror surface dimension and tiltation is less than 2.5 degrees about the coordinate axis.

In the last example, genetic algorithms (GA) were used to design a spherical-surface GRIN lens laser beam shaping system, where catalog GRIN glass types are used and the number of lens element required is an optimization variable. The optical design for this system involves maximizing a merit function based on output beam irradiance, size, and direction of propagation within a 26-dimensional parameter space. To solve this optical design problem, the GA optimized surface shapes of the GRIN elements and their spacing, determined the actual number of GRIN elements in the solution, up to a certain limit (four, in this case), and the type of GRIN material for each element was selected from a GRIN glass catalog. These applications show that the geometrical methods for design of range of laser beam shaping systems are effective for these types of configurations.

\section{REFERENCES}

1. A.C. Claus, "On Archimedes' Burning Glass," Appl. Opt. 12.10, p. A14, 1973.

2. O.N. Stavroudis, “Comments On: On Archimedes’ Burning Glass," Appl. Opt. 12.10, p. A16, 1973.

3. M.H. Cobble, "Theoretical Concentration for Solar Furnaces," Solar Energy 5.2, p. 61, 1961.

4. O. Kamada, "Theoretical Concentration and Attainable Temperature in Solar Furnaces," Solar Energy 9.1, pp. 39-47, 1965.

5. K.-E. Hassan and M.F. El-Refaie, "Theoretical Performance of Cylindrical Parabolic Solar Concentrators," Solar Energy 15, pp. 219-244, 1973.

6. R.A. Zakhidov and A.A. Vainer, "Distribution of Radiation Produced by a Paraboloidal Concentrator," Geliotekhnika (Applied Solar Energy) 10.3, pp. 34-40, 1974. The Russian to English translation of this article was part of the Allerton Press Journal Program.

7. R.F. Jones Jr., "Collection properties of generalized light concentrators," J. Opt. Soc. Am. 67.11, pp. 1594-1598, 1977.

8. W.T. Welford and R. Winston, High Collection Nonimaging Optics, Academic Press, New York, 1989.

9. R. Winston, "Principles of Solar Concentrators of a Novel Design," Solar Energy 16, pp. 89-95, 1974.

10. R. Winston and H. Hinterberger, "Principles of Cylindrical Concentrators for Solar Energy," Solar Energy 17, pp. $255-$ $258,1975$.

11. D.G. Burkhard and D.L. Shealy, "Design of Reflectors Which Will Distribute Sunlight in a Specified Manner," Solar Energy 17.1, pp. 221-227, 1975.

12. J.H. McDermit, "Curved reflective surfaces for obtaining prescribed irradiation distributions," Ph.D. Dissertation, University of Mississippi, 1972.

13. T.E. Horton and J.H. McDermit, "Optical Design of Solar Concentrators," J. Energy 4.1, pp. 4-9, 1980.

14. D.L. Shealy and H.M. Berg, "Simulation of optical coupling from surface emitting LEDs," Appl. Opt. 22.11, pp. 1722-1730, 1983.

15. D.R. Gabardi and D.L. Shealy, "Coupling of domed light-emitting diodes with a multimode step-index optical fiber," Appl. Opt. 25.19, pp. 3435-3442 (1986). 
16. J.H. McDermit and T.E. Horton, "Reflective optics for obtaining prescribed irradiative distributions from collimated sources," Appl. Opt. 13.6, pp. $1444-1450,1974$.

17. D.G. Burkhard, D.L. Shealy and R.U. Sexl, "Specular Reflection of Heat Radiation from an Arbitrary Reflector Surface to an Arbitrary Receiver Surface," Int. J. Heat Mass Transfer 16, pp. 271-280, 1973.

18. D.G. Burkhard and D.L. Shealy, "View Function in Generalized Curvilinear Coordinates for Specular Reflection of Radiation from a Curved Surface," Int. J. Heat Mass Transfer 16, pp. 1492-1496, 1973.

19. J.S. Schruben, "Analysis of rotationally symmetric reflectors for illuminating systems," J. Opt. Soc. Am. 64.1, pp. 55-58, 1974.

20. D.G. Burkhard and D.L. Shealy, "Specular aspheric surface to obtain a specified irradiance from discrete or continuour line source radiation: design," Appl. Opt. 14.6, pp. 1279-1284, 1975.

21. D.G. Burkhard and D.L. Shealy, "A Different Approach to Lighting and Imaging: Formulas for Flux Density, Exact Lens and Mirror Equations, and Caustic Surfaces in Terms of the Differential Geometry of Surfaces," in Materials and Optics for Solar Energy Conversion and Advanced Lighting Technology, Carl. M. Lampert and Sandor Holly, eds., Proc. SPIE 692, pp. 248-272, 1986.

22. J.M. Gordon and A. Rabl, "Reflectors for uniform far-field irradiance: fundamental limits and example of an axisymmetric solution," Appl. Opt. 37.1, pp. 44-47, 1998.

23. B.S. Westcott and A.P. Norris, "Reflector synthesis for generalized far-fields," J. Phys. A: Math. Gen. 8.4, pp. 521-532, 1975 .

24. F. Brickell and B.S. Westcott, "Reflector design for two-variable beam shaping in the hyperbolic case," J. Phys. A: Math. Gen. 9.1, pp. 113-128, 1976.

25. J. H. McDermit and T. E. Horton, "Reflective optics for obtaining prescribed irradiative distributions from collimated sources," Appl. Opt. 13, pp. 1444-1450, 1974.

26. P. W. Malyak, "Two-mirror unobscured optical system for reshaping the irradiance distribution of a laser beam," Appl. Opt. 31, pp. 4377-4383, 1992.

27. D. L. Shealy and S. H. Chao, "Design and analysis of an elliptical Gaussian laser beam shaping system," in Laser Beam Shaping II, Fred M. Dickey, Scott C. Holswade, and David L. Shealy, eds., Proc. SPIE 4443, pp. 24-35, 2001.

28. B. R. Frieden, "Lossless conversion of a plane laser wave to a plane wave of uniform irradiance," Appl. Opt. 4.11, pp. 1400-1403, 1965.

29. J. L. Kreuzer, "Coherent light optical system yielding an output beam of desired intensity distribution at a desired equiphase surface," U. S. Patent 3,476,463, 4 November, 1969

30. P. W. Rhodes and D. L. Shealy, "Refractive optical systems for irradiance redistribution of collimated radiation: their design and analysis," Appl..Opt. 19, pp. 3545-3553, 1980.

31. P. W. Rhodes, "Design and analysis of refractive optical systems for irradiance redistribution of collimated radiation," M. S. Thesis, The University of Alabama in Birmingham, 1979.

32. J. A. Haffnagle and C. M. Jefferson, "Design and performance of a refractive optical system that converts Gaussian to a flattop beam," Appl. Opt. 19, pp. 5488-5499, 2000.

33. W. Jiang, D. L. Shealy, and J. C. Martin, "Design and testing of a refractive reshaping system," in Current Developments in Optical Design and Optical Engineering III, Robert E. Fisher and Warren J. Smith, eds., Proc. SPIE 2000, pp. 64-75, 1993.

34. W. Jiang, "Application of a laser beam profile reshaper to enhance performance of holographic projection systems," Ph.D. Dissertation, The University of Alabama at Birmingham, 1993.

35. W. Jiang, D.L. Shealy, and K.M. Baker, "Optical design and testing of a holographic projection system," in Diffractive and Holographic Optics Technology, I Cindrich and S. H. Lee, eds., Proc. SPIE 2152, pp. 244-252, 1994.

36. Laser Beam Shaping, Fred M. Dickey and Scott C. Holswade, eds., Proc. SPIE 4095, 2000.

37. Laser Beam Shaping: Theory and Techniques, Fred M. Dickey and Scott C. Holswade, eds., Marcel Dekker, Inc., New York, 2000.

38. Laser Beam Shaping II, Fred M. Dickey and Scott C. Holswade, and David L. Shealy, eds., Proc. SPIE 4443, 2001.

39. Chapter 1, pp. 12-14, Ref. 37.

40. D. L. Shealy, "Geometrical Methods," in Laser Beam Shaping: Theory and Techniques, Fred M. Dickey and Scott C. Holswade, eds., pp. 163-213, Marcel Dekker, Inc., New York, 2000.

41. D. L. Shealy, "Theory of geometrical methods for design of laser beam shaping systems, in Laser Beam Shaping, Fred M. Dickey and Scott C. Holswade, eds., Proc. SPIE 4095, pp. 1-15, 2000. 
42. N. C. Evans and D. L. Shealy, "Optimization-based techniques for laser shaping optics," in Laser Beam Shaping: Theory and Techniques, Fred M. Dickey and Scott C. Holswade, eds., Chapt. 5, Marcel Dekker, New York, 2000.

43. S. Solimeno, B. Crosignani, and P. DiPorto, Guiding, Diffraction, and Confinement of Optical Radiation, p. 49, Academic Press, Orlando, 1984.

44. A.K. Ghatak and K. Thyagarajan, Contemporary Optics, p. 24, Plenum Press, New York, 1980.

45. D.G. Koch, "Simplified Irradiance/Illuminance Calculations in Optical Systems," presented at the International Symposium on Optical Systems Design, Berlin, Germany, September 14, 1992, and published in Proc. SPIE 1780-14, 1992.

46. D.G. Burkhard, and D.L. Shealy, "Simplified formula for the illuminance in an optical system," Appl. Opt. 20, pp. 897909, 1981.

47. M. Born and E. Wolf, Principles of Optics, 5th Edition, Pergamon Press, New York, 2000, p. 115.

48. P. Mouroulis and J. Macdonald, Geometrical Optics and Optical Design, Oxford University Press, New York, 1997.

49. D.L. Shealy and D.G. Burkhand, "Analytical illuminance calculation in a multi-interface optical system," Opt. Act. 22.6, pp. 485-501, 1975.

50. D.L. Shealy and D.G. Burkhand, "Flux density for ray propagation in discrete index media expressed in terms of the intrinsic geometry of the deflecting surface," Opt. Act. 20.4, pp. 287-301, 1973.

51. Ealing Electro-Optics, Product Guide, K-13, Holliston, MA, 1989.

52. W.L. Wolf, G.J. Zissis, eds, p. 7-83, The Infrared Handbook, revised edition, Environmental Research Institution of Michigan, Ann Arbor, MI, 1989.

53. D. Malacara, Optical Shop Testing, pp. 283, John Wiley and Sons, Inc., New York (1977).

54. W. Jaing and D.L. Shealy, "Development and testing of a refractive laser beam shaping system," in Laser Beam Shaping, Fred M. Dickey and Scott C. Holswade, eds, Proc. SPIE 4095, pp.165-175, 2000.

55. Born and Wolf, section 4.3.

56. D. F. Cornwell, "Non-projective transformations in optics," Ph.D. Dissertation, University of Miami, 1980.

57. D. F. Cornwell, "Nonprojective transformations in optics," Proc. SPIE 294, pp. 62-72, 1981.

58. Focus Software, Inc., P.O. Box 18228, Tucson, AZ 85731.

59. C. Wang and D. L. Shealy, "Design of gradient-index lens system for laser beam reshaping," Appl. Opt. 32, pp. 47634769, 1993.

60. N. C. Evans and D. L. Shealy, "Design of a gradient-index beam shaping system via a genetic algorithm optimization method," in Laser Beam Shaping, F.M. Dickey and S.C. Holswade, eds., Proc. SPIE 4095, pp. 27-39, 2000.

61. N.C. Evans, "Genetic Algorithm Optimization Methods in Geometrical Optics," Ph.D. Dissertation, The University of Alabama at Birmingham, 1999.

62. K.M. Baker, D.L. Shealy, and W. Jiang, "Directional light filters: three-dimensional azo dye formed images within optical resins," Proc. SPIE 2404, pp. 144-158 (1995).

63. K.M. Baker, "Highly corrected submicrometer grid patterning on curved surfaces," Appl. Opt. 38, pp. 339-351, 1999.

64. K.M. Baker, "Highly corrected close-packed microlens arrays and moth-eye structuring on curved surfaces," Appl. Opt. 38, pp. 352-356, 1999. 